\title{
NUEVA COMUNICACIÓN SONORA. CARTOGRAFÍA, GRAMÁTICA Y NARRATIVA TRANSMEDIA DEL PODCASTING
}

\author{
New sound communication. Cartography, grammar \\ and transmedia narrative of podcasting
}

\section{David García-Marín y Roberto Aparici}

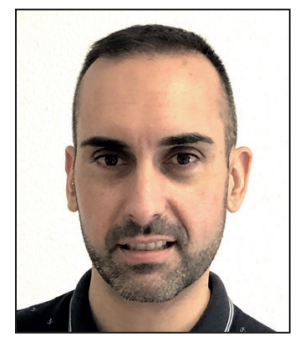

David García-Marín es licenciado en Periodismo por la Universidad Complutense de Madrid, master de Radio en la Universidad San Jorge de Zaragoza, y master de Comunicación y Educación en la Red de UNED. Investigador doctorando en Sociología (rama medios de comunicación) en el programa de Cambio Social en Sociedades Contemporáneas por la UNED. Profesor del Master de Comunicación y Educación en la Red y del Master de Periodismo Transmedia UNED-EFE, es miembro del grupo de investigación Análisis de pedagogías digitales: Comunicación, redes sociales y nuevas narrativas (UNED) y miembro de la Red $1+D$ de Educación Mediática.

https://orcid.org/0000-0002-4575-1911

dgarciamarin@invi.uned.es

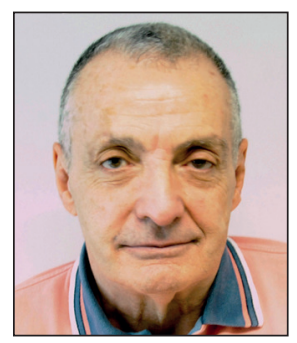

Roberto Aparici es profesor titular de Comunicación y Educación del Departamento de Didáctica, Organización Escolar y Didácticas Especiales de la UNED. Director del Master de Comunicación y Educación en la Red y del Master de Periodismo Transmedia de UNED y la agencia EFE. Investigador en educomunicación, educación digital interactiva, nuevas narrativas, cultura de la participación y transalfabetizaciones. Investigador principal de la Red I+D de Educación Mediática. https://orcid.org/0000-0002-1908-1029

raparici@edu.uned.es

Universidad Nacional de Educación a Distancia. Facultad de Educación Departamento de Didáctica, Organización Escolar y Didácticas Especiales Juan del Rosal, 14. 28040 Madrid, España

\section{Resumen}

Tras década y media de desarrollo, el podcasting ha construido una narrativa propia que provoca una ruptura del lenguaje tradicional de la radio al basar sus dinámicas narrativas tanto en la configuración transmedia de sus contenidos como en las estrategias multiplataforma. Mediante la aplicación de técnicas cualitativas de análisis de los podcasts españoles más relevantes y la realización de entrevistas en profundidad semiestructuradas a informadores clave, esta investigación presenta una cartografía de las diversas plataformas y distintos contenidos que los podcasters utilizan para expandir sus relatos más allá del lenguaje sonoro. Los resultados muestran que el podcasting ofrece sus mensajes en un amplio rango de soportes tanto digitales como analógicos que se complementan funcionalmente y que exigen producir materiales en lenguajes mediáticos diferenciados. Asimismo, los podcasts presentan una gran variedad de contenidos que, lejos de comportarse de forma aislada, conforman una gramática lógica y coherente.

\section{Palabras clave}

Podcasts; Podcasting; Medios digitales; Narrativa digital; Transmedia; Radio; Hipermedia; Convergencia; Medios interactivos; Lenguaje sonoro.

\begin{abstract}
Podcasting has created an own narrative that provokes a rupture of the traditional radio's language due to the transmedia configuration of its contents and its multiplatform strategies. By analyzing the most relevant Spanish podcasts and through in-depth interviews, this investigation presents a cartography of the diverse platforms and the different contents that podcasters use so as to expand their stories beyond the sound language. Our results demonstrate that podcasting offers its messages in a wide range of platforms, digital and analog, that functionally complement each other. This involves producing materials in different media language. Moreover, podcasting presents a great variety of contents that, instead behaving in an isolated way, conform a logical and coherent grammar.
\end{abstract}

\section{Keywords}

Podcasts; Podcasting; Digital media; Digital storytelling; Transmedia; Radio; Hypermedia; Convergence; Interactive media; Sound language. 
García-Marín, David; Aparici, Roberto (2018). “Nueva comunicación sonora. Cartografía, gramática y narrativa transmedia del podcasting". El profesional de la información, v. 27, n. 5, pp. 1071-1081.

https://doi.org/10.3145/epi.2018.sep.11

\section{Introducción}

El advenimiento y la popularización de internet ha provocado en las dos últimas décadas la aparición de un nuevo ecosistema de la comunicación sonora, dando paso a nuevos conceptos, como ciberradio (Cebrián-Herreros, 2009) o post-radio (Ortiz-Sobrino, 2012), y nuevos formatos que desbordan las dinámicas propias de la radio analógica del siglo XX.

La comunicación sonora de masas llegó a la Red cuando las grandes emisoras clásicas comenzaron a utilizar internet como punto de acceso diferenciado a la misma programación que ya emitían vía hertziana, de forma que la Web funcionaba como repetidor digital de los contenidos.

En una segunda fase de desarrollo de la radio digital, los portales que soportaban la emisión online de estas grandes emisoras complementaron sus contenidos con elementos informativos construidos en lenguajes diferentes al sonoro (imágenes, infografías, etc.) y abrieron espacios de participación del usuario mediante la inclusión de comentarios. Paralelamente, nacieron numerosos proyectos de radio exclusivamente digital, sin ningún tipo de soporte hertziano.

La llegada de la radio digital a la carta, que posibilita la descarga y escucha asíncrona de contenidos, constituye el siguiente paso en la evolución del audio online. Este modelo quiebra la temporalidad clásica de la radio y ofrece una mayor dosis de personalización de la escucha a un oyente que ya es capaz de generar su propia parrilla de programación y adaptar el consumo a sus hábitos, disponibilidad e intereses. La radio a la carta ofrece también la posibilidad de consumir contenidos sin necesidad de conexión a la Red mediante la descarga de programas para su almacenamiento en los dispositivos de reproducción disponibles en el mercado (ordenadores, tabletas, teléfonos inteligentes, reproductores de $\mathrm{mp3}$, etc.).

El paso decisivo para la aparición del podcasting fue la introducción de los mecanismos de sindicación que permiten la suscripción a los contenidos sonoros para su descarga automática. La tecnología requerida para la utilización del podcasting fue creada en 2004 en Estados Unidos por Adam Curry, un antiguo vídeo jockey del canal televisivo MTV, en colaboración con Dave Winer, uno de los desarrolladores del motor de sindicación de contenidos RSS. Ambos fueron capaces de construir un método de emisión de audio online que pone el archivo sonoro a disposición de cualquier oyente para ser escuchado a su conveniencia. El 10 de agosto de 2004, la revista especializada en tecnología Wired afirmaba que la posibilidad de suscripción que permite el podcasting constituye una gran novedad al ofrecer nuevas oportunidades de consumo e interacción con el audio más allá de las ofrecidas por la radio tradicional.

De este modo, el podcasting se configuró como un medio de comunicación que surgió de la fusión de dos tecnologías previamente implantadas:

- el audio digital;

- los motores de descarga automática de contenidos.

Esta unión

"permite la distribución y recepción automatizada de archivos de audio comprimido tras una suscripción por parte del usuario" (Gallego-Pérez, 2010).

Massarelli y Perrotta (2006) consideran el podcasting como una tecnología desafiante para los medios tradicionales, que permite a los oyentes crear su propio contenido, a la vez que constituye un cambio en los hábitos de escucha de la radio convencional. Por su parte, Berry (2016) reflexiona acerca del papel que juega el podcasting dentro del ecosistema mediático y plantea el debate sobre si esta práctica comunicativa constituye un nuevo medio per se o constituye la última fase evolutiva de un medio cambiante y adaptable como la radio.

\section{El paso decisivo para la aparición del} podcasting fue la introducción de los mecanismos de sindicación que permiten la suscripción a los contenidos sonoros y, por tanto, su descarga automática

McHugh (2016) ha centrado sus investigaciones en el crecimiento de las redes de podcasts independientes y las diferencias percibidas entre la radio y el podcasting como modos de distribución y recepción de contenidos sonoros. Este autor defiende que el podcasting está fomentando un género novedoso y más informal de narrativa sonora centrado en unas fuertes relaciones entre los presentadores y los oyentes, con contenidos más improvisados y menos elaborados. Esta concepción dialoga de cerca con la visión de la nueva radio definida por Dubber (2013) como una "ecología de fenómenos relacionados, pero diferentes".

Tras casi década y media de desarrollo del medio, los creadores de podcasts (podcasters) han iniciado un proceso de sofisticación de la estructura narrativa del podcasting desbordando su lenguaje inicial -exclusivamente sonoro- que copiaba los antiguos formatos de la radio. Los podcasts han adquirido una gramática narrativa propia, autónoma, específica y separada de la radio que se adentra en experiencias creativas transmedia al extender sus relatos en plataformas diversas, construir diferentes tipos de contenidos en distintos lenguajes mediáticos e incluir a los oyentes en la producción de los programas (García-Marín, 2017).

\section{Objetivo y metodología}

Esta investigación pretende analizar las estrategias narrativas utilizadas por los podcasters en España a partir del estudio de dos dimensiones: 
Tabla 1. De la radio al podcast. Fases evolutivas del medio radiofónico hasta la aparición del podcasting. Elaborado a partir de Cebrián-Herreros (2009).

\begin{tabular}{|l|l|}
\hline Fase 1. Período pre-digital & Radio exclusivamente hertziana. \\
\hline Fase 2. Llegada a la Web & Las emisoras usan internet como repetidor digital vía streaming. \\
\hline Fase 3. Desarrollo de la radio digital & $\begin{array}{l}\text { Enriquecimiento de los portales online de las radios digitales con la inserción de elementos informativos com- } \\
\text { plementarios (imágenes, infografías, posibilidad de realizar comentarios...). Llegan las radios exclusivamente } \\
\text { digitales, sin soporte hertziano. }\end{array}$ \\
\hline Fase 4. Aumento de la interactividad & Empaquetado de programas. Posibilidad de escucha asíncrona y descarga. Llega la radio digital a la carta. \\
\hline Fase 5. La sindicación & $\begin{array}{l}\text { Se introduce la descarga automatizada de programas para su escucha offline mediante motores RSS. Aparece } \\
\text { el podcasting. }\end{array}$ \\
\hline
\end{tabular}

- dispersión de sus relatos en diferentes plataformas online y offline;

- producción de diferentes categorías de contenidos que expanden el mensaje ofrecido en sus programas.

Se establecerá una cartografía de las plataformas y los servicios que utiliza el medio y una taxonomía de sus diferentes textualidades.

Desde su creación, el podcasting ha evolucionado en dos direcciones (Bonini, 2015):

- creación amateur sin ánimo de lucro;

- uso comercial en busca de beneficios económicos.

A pesar de la existencia de varios ejemplos de podcasts profesionales vinculados a grandes empresas mediáticas, en el panorama español la mayoría de proyectos de podcasting son elaborados de forma amateur e independiente (Aparici; García-Marín, 2018). Por este motivo, enfocamos nuestro trabajo de campo, ejecutado en los meses de octubre a diciembre de 2017, en el podcasting no profesional donde se establece una mayor libertad y riqueza expresiva y unas mayores dosis de experimentación narrativa. Nuestra metodología de trabajo tuvo un carácter cualitativo mediante el uso de dos instrumentos de recolección de datos:

- entrevistas semiestructuradas a podcasters y oyentes;

- análisis documental aplicado a una selección de podcasts del ámbito español.

Rosado-Millán, García-García y Kaplún-Hirsz (2014) conciben el análisis documental como el estudio de aquellas informaciones registradas sobre un soporte que proporcionan un conocimiento útil,

"usadas cuando se pretende obtener información general o especializada acerca de un tema determinado".

Para trabajar con esta técnica, se construyó una ficha de análisis con 60 indicadores que fue aplicada a los 11 programas ganadores de la última edición de los Premios Nacionales de Podcasting, entregados en octubre de 2017. De los 60 indicadores, aquí contemplamos exclusivamente los que tienen relación con la narrativa de los podcasts (tabla 2). El objetivo fue registrar el número de plataformas utilizadas por cada podcast y las categorías textuales generadas por cada programa. Para estudiar estas categorías textuales utilizamos el modelo de Askwith (2007), que divide los contenidos de los universos transmedia en la siguiente taxonomía:

- accesos expandidos;
- contenidos adaptados;

- contenidos expandidos;

- actividades relacionadas;

- productos de la marca.

Los 11 programas analizados fueron (entre paréntesis, la categoría a la que pertenece cada uno):

- La morsa era yo (Arte y/o cultura general);

- Guiller y yo (Bitácora personal);

- Ohhh TV! Podcast (Cine, series y/o televisión);

- Desde boxes (Deporte o actividad física);

- Perspectiva (Economía o negocios);

- Ya conoces las noticias (General);

- Con todos mis respetos (Humor);

- Por qué podcast (Multitemática);

- Bitácora de ciberseguridad (Revelación);

- Crónica en negro (Sociedad);

- Esto con Jobs no pasaba (Tecnología).

De forma paralela se llevaron a cabo 22 entrevistas cualitativas semiestructuradas a informadores clave. Corbetta (2007) define este método como una conversación provocada por el entrevistador,

"realizada a sujetos seleccionados a partir de un plan de investigación, en un número considerable, que tiene una finalidad de tipo cognitivo, guiada por el entrevistador, y con un esquema de preguntas flexible y no estandarizado" (Corbetta, 2007).

Los sujetos entrevistados se dividieron en las siguientes categorías:

- pioneros del medio y representantes de asociaciones nacionales de podcasting (cinco entrevistados);

- podcasters en activo (diez entrevistados);

- oyentes (siete entrevistas).

Los criterios de selección de los podcasters participantes en la muestra fueron su relevancia y visibilidad en la comunidad y la producción de podcasts con frecuentes actualizaciones (diarias, semanales o quincenales).

Los oyentes fueron elegidos mediante muestreo aleatorio simple utilizando el canal de Telegram Comunidad Podkas, que reúne a unos 200 entusiastas del medio.

Los sujetos entrevistados fueron:

- José Antonio Gelado: primer podcaster español;

- Daniel Aragay: pionero del podcasting en España;

- Sebas Oliva: fundador y primer presidente de la Asociación Podcast (2010); 
- Agustín Palmeiro: presidente de la Asociación Podcast en 2017 y 2018;

- Raúl de la Puente: vicepresidente de la Asociación de Escuchas de Podcasting (Asespod);

- Eduardo Nordmann: podcaster, autor de varios programas sobre filosofía y humor;

- Dumakae: podcaster especializada en series y televisión. Cuenta con varios premios nacionales;

- Tamara León: creadora de programas de actualidad política y social;

- Blanca Santamaría: podcaster en el programa de tertulia Por qué podcast, espacio con diversos premios nacionales;

- Francisco Portillo: autor del podcast de humor Condenados, ganador de numerosos premios;

- Emilio Cano: podcaster fundador de la red Emilcar FM. Ganador del premio al Mejor Podcaster Masculino de España en varias ediciones;

- Goyo Salduero: fundador y director del podcast de temática histórica Histocast;

- Juan Ortiz: podcaster en el programa sobre arquitectura La morsa era yo;

- Esteban Pérez: presentador del programa de historia Zafarrancho podcast;

- Carmen Moreno: podcaster en el programa Carmenia en Dallas.

No incluimos el nombre de los siete oyentes entrevistados, al haberles garantizado el anonimato a la hora de publicar los resultados de la investigación.

Para la producción de datos e interpretación de resultados, ambas técnicas de investigación (análisis documental y entrevistas) se articularon por complementación, debido a que ambas ofrecen perspectivas diferentes sobre el mismo objeto de estudio (Callejo; Viedma, 2005). Siguiendo la taxonomía propuesta por Scolari (2008) a propósito de los diferentes paradigmas teóricos útiles para el estudio de los nuevos medios, ubicamos esta investigación en un campo teórico híbrido que bebe tanto del paradigma interpretativo-cultural como del semiótico-discursivo.

\section{Cartografía de las plataformas}

Para la construcción del mapa de plataformas, recurrimos a los datos producidos tanto por las entrevistas como por la aplicación de las fichas de análisis documental. Tras la ejecución del trabajo de campo, observamos que un total de siete categorías (plataformas) componen el mapa estructural del podcasting. Mostramos su análisis a continuación.

\subsection{Repositorios y podcat- chers}

El punto de partida de todo proyecto de podcasting es la contenidos) del podcasting. creación de un programa en formato sonoro que se aloja online y que puede ser escuchado en streaming o bajo suscripción mediante motores de sindicación. El alojamiento se realiza en las plataformas o repositorios de podcasts disponibles en el mercado (iVoox, iTunes y Spreaker son los más utilizados en España). Estos servicios permiten que los podcasters inicien la construcción de una interesante comunidad alrededor de su trabajo al presentar espacios para la inserción de comentarios y ofrecer posibilidades para la difusión de cada capítulo mediante enlazado de url o inserción del código html.

Los podcasts han adquirido una gramática narrativa propia, autónoma y especifica que se adentra en experiencias creativas transmedia al extender sus relatos en plataformas diversas y construir diferentes tipos de contenidos

El consumo en movilidad que los usuarios suelen realizar en este medio determina que el acceso de los oyentes a los proyectos de podcasting se produzca de forma mayoritaria mediante las aplicaciones para teléfonos móviles de escucha y descarga, los podcatchers, que consisten en pequeñas aplicaciones informáticas para la suscripción, descarga automática, gestión y consumo de podcasts. En 2017 y 2018, los podcatchers más utilizados en la podcastfera española son: iVoox, Overcast, Castro, Podcasts (Apple), Spreaker, TwitBlogCast, Downcast, Ucast, Pocket Casts, Podcast Addict y Podcast Republic. Estas aplicaciones asumen un papel esencial en la escucha de podcasts, tal y como afirma la mayoría de los oyentes entrevistados:

“Mi consumo es al 99\% desde el móvil y a través de podcatcher" (oyente 4).

Tabla 2. Ficha de análisis de las características transmedia (dispersión en plataformas y taxonomía de

\begin{tabular}{|c|c|}
\hline Plataformas & Contenidos \\
\hline $\begin{array}{l}\text { Número de páginas web/blogs del podcast. ¿Tienen } \\
\text { dominio propio o pertenecen a terceros (Blogger, Wor- } \\
\text { dpress, Wix, etc.)? }\end{array}$ & Accesos expandidos \\
\hline $\begin{array}{l}\text { ¿Presencia en servicios de podcasting: iVoox, iTunes, } \\
\text { Spreaker...? }\end{array}$ & $\begin{array}{l}\text { Interacciones sociales. Pueden ser: } \\
\text {-relación horizontal } \\
\text {-relación vertical } \\
\text {-relación diagonal }\end{array}$ \\
\hline ¿Presencia en redes sociales? ¿Cuáles? & Contenidos adaptados \\
\hline ¿Presencia en radio convencional? ¿En radio digital? & $\begin{array}{l}\text { Contenidos expandidos. Pueden ser: } \\
\text {-extensión textual } \\
\text {-información relevante } \\
\text {-información extratextual }\end{array}$ \\
\hline \multirow{2}{*}{$\begin{array}{l}\text { ¿Distribuye su relato en entornos diferentes al ciberes- } \\
\text { pacio? ¿En cuáles? ¿Presenta objetos extradigitales } \\
\text { (merchandising, etc.? }\end{array}$} & Productos de la marca \\
\hline & $\begin{array}{l}\text { Actividades relacionadas. De cuatro tipos: } \\
\text {-temáticas } \\
\text {-experienciales } \\
\text {-productivas } \\
\text {-competitivas }\end{array}$ \\
\hline
\end{tabular}


"Siempre escucho por podcatchers, además soy de las que va cambiando de uno a otro según me vaya gustando más uno u otro" (oyente 5 ).

"Normalmente uso podcatchers. Yo utilizo Pocket Casts, pero habitualmente primero me suscribo por iVoox y luego lo busco en Pocket Casts, porque esta aplicación va mucho mejor" (oyente 6).

\section{Numerosos programas divulgativos pre- sentan en sus webs o blogs abundante material en formato audiovisual a fin de complementar los relatos que, por su ca- rácter exclusivamente sonoro, plantean dificultades de recepción o comprensión}

\subsection{Web/blog}

La inmensa mayoría de los podcasts que podemos encontrar en el ámbito español utilizan algún tipo de página online, con especial predominancia del formato blog. Esta plataforma otorga personalidad propia a los podcasts y les permite diferenciarse de otros proyectos. El tipo de sitio web o blog- utilizado guarda relación con la temática que trata el programa y depende de si éste se encuentra sujeto a determinados eventos de actualidad. En el caso de los espacios intemporales, la explotación del formato web se considera una estrategia más acertada, útil y efectiva que la construcción de un blog. Al contrario, aquellos proyectos que tienen una mayor atadura a la actualidad y un período de actualización más corto (diario o semanal) tienden a usar el formato blog, al disponer sus contenidos en orden inversamente cronológico de modo que el texto que primero encuentra el usuario es el último en ser publicado y, además, suele tener vinculación con el último episodio del podcast lanzado. Juan Ortiz lo expresa del siguiente modo: en sus webs o blogs abundante y rico material en formato fotográfico y audiovisual a fin de complementar aquellos relatos que, por su carácter exclusivamente sonoro, plantean dificultades de recepción o comprensión. Por ejemplo, determinados podcasts de temática histórica introducen mapas para guiar al usuario en su recorrido por la descripción de los avatares que narra el episodio en formato sonoro. Gracias a estos contenidos fotográficos y audiovisuales, los escuchas pueden encontrar informaciones extra que no son tratadas en el podcast o, al menos, no con la profundidad que proporcionan los medios visuales. Afirma Goyo Salduero:

"En la web tenemos tanto imágenes, algunas fotos, como los mapas que, por supuesto, son clicables y ampliables".

Las webs o blogs sirven también como principal puerta de acceso a los proyectos de podcasting, especialmente en el caso de los usuarios menos avezados con las plataformas de podcasts y los dispositivos móviles, y que encuentran una mayor facilidad en el dominio de los ordenadores de escritorio o portátiles y los buscadores online tradicionales. La obligación de descargar una aplicación específica de escucha y suscripción de podcasts, la búsqueda del programa que se quiere escuchar en tal aplicación, el hecho de suscribirse para recibir los nuevos episodios de forma automática y la actualización periódica de la lista de programas descargados pueden comprender acciones demasiado complejas para los escuchas con menos habilidades tecnológicas. Además, la web/blog sirve como espacio para el alojamiento de comentarios de los seguidores sobre los contenidos creados por los podcasters.

Asimismo, esta plataforma es utilizada por los oyentes como un espacio destinado a la profundización en el conocimiento del proyecto y su temática para aquellos usuarios más comprometidos con la marca:

"Consulto las webs porque cuando ya te gusta bastante
"El formato-web o blog- depende de los contenidos del podcast, así vendrá bien de una manera u otra. Para eso, es fundamental la temporalidad, si un podcast vive o no del último episodio".

Estos espacios -web y blog- tienen como principal función la reunión de los contenidos que construyen la trama narrativa del proyecto en claras lógicas hipermedia al situar textualidades construidas en diferentes lenguajes mediáticos y amalgamadas entre sí mediante hiperenlaces (Moreno, 2012):

"Hicimos el blog, principalmente, por aunar los contenidos de nuestro podcast" (Carmen Moreno)

Al margen de esta labor de convergencia mediática (Jenkins, 2006), estas plataformas adquieren un claro valor explicativo de los contenidos que se narran en el podcast, completando la experiencia del usuario. Numerosos programas divulgativos presentan

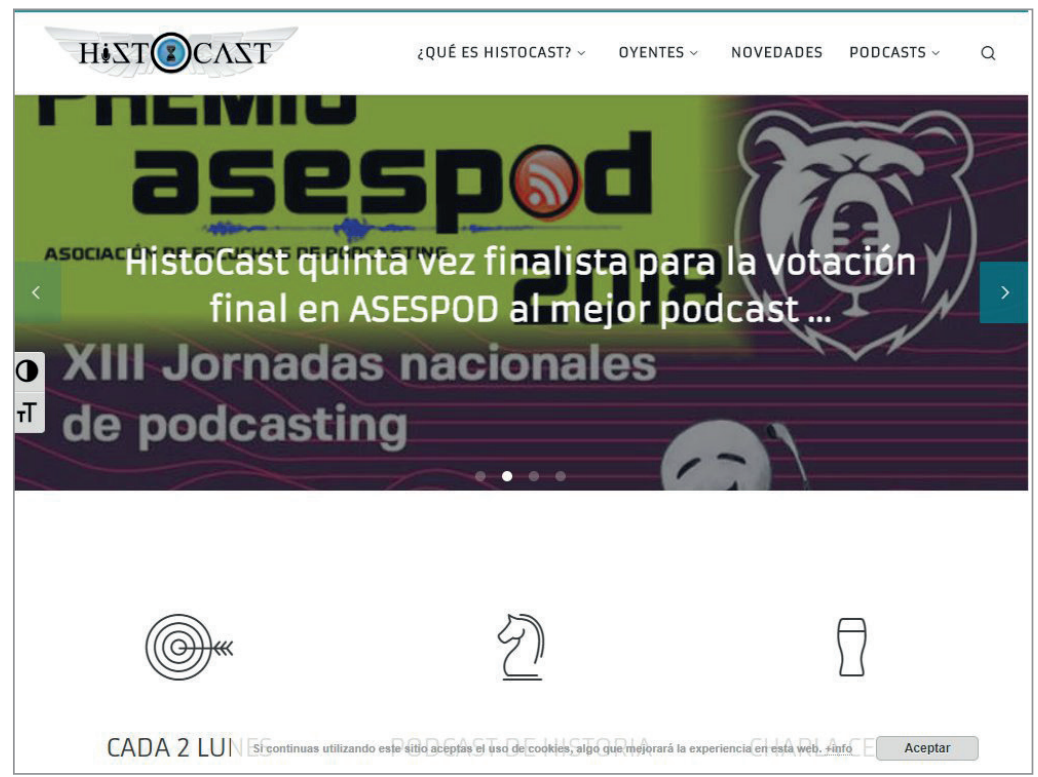

Web del podcast Histocast, uno de los más relevantes del panorama independiente en lengua hispana https://www.histocast.com 
un podcast, quieres saber quién está detrás del micrófono, cuánto tiempo llevan publicando esos podcasters, etc." (oyente 3 ).

\subsection{Redes sociales}

Fundamentales en el podcasting, las redes sociales -Twitter y Facebook son las más comunes en el medio- constituyen soportes para promocionar los programas, así como para proporcionar avisos sobre las noticias relacionadas con los proyectos (sobre todo, para anunciar el lanzamiento de nuevos capítulos) e interactuar y dialogar con los fans. Las redes sociales sirven para recoger las aportaciones de un tipo de usuario menos comprometido o con un discurso menos elaborado y que, por tanto, rechaza realizar comentarios en los blogs o webs. Así lo explica Emilio Cano:

"Las redes sociales son fundamentales para el diálogo, para recibir esa parte de feedback de ese oyente más perezoso que no le apetece irse al blog o que tampoco tiene tanto que decir".

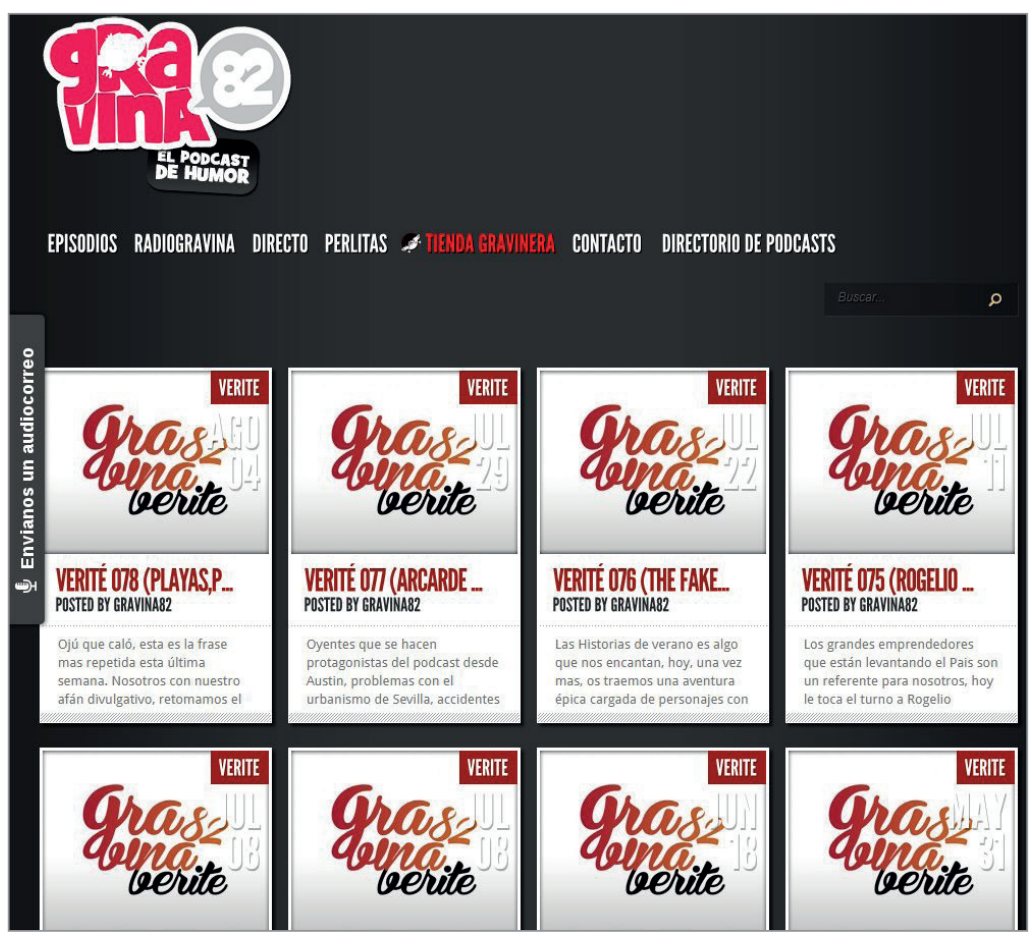

Web del podcast de humor Gravina 82, que hace un uso intensivo de las extensiones mediante lenguajes audiovisuales

https://gravina82.com
Los oyentes perciben las redes sociales en el podcasting como un canal directo de interacción con sus creadores favoritos. En este espacio gobiernan la cercanía y la facilidad de acceso al podcaster y la comunicación horizontal con otros fans del programa:

"En Twiter, el oyente se siente con mayor soltura a la hora de expresar sus opiniones. Es muy útil para hablar con gente que escucha las mismas cosas que tú" (oyente 5).

La relación entre creadores y escuchas a través de las redes sociales puede derivar en la construcción de contenido significativo, incrementándose así la participación del usuario que puede potencialmente convertirse en coautor de los relatos

La relación entre creadores y escuchas a través de estos instrumentos sociales puede derivar, ocasionalmente, en la construcción de contenido significativo, incrementándose así la participación del oyente que en determinadas circunstancias puede potencialmente convertirse en coautor de los relatos:

"Nosotros creamos una cosa que eran los retos para Carmenia. Cualquiera podía escribir por las redes y proponer un desafío que creaba significados y contenidos nuevos" (Carmen Moreno).

"Es muy raro que te dejen un comentario en el blog. Tienes que estar en las redes sociales, ya que ahí de vez en cuando se generan conversaciones que se usan en los podcasts" (Juan Ortiz).

En los últimos años, los podcasters han asumido paulatina- mente el uso de Telegram como espacio para interactuar con sus seguidores más fieles de forma más privada y cerrada. Gracias a estos chats específicos, cada proyecto construye y centraliza su propia comunidad dentro de la podcastfera. El intercambio de materiales y conocimientos significativos vinculados a la temática del podcast entre miembros de estas microcomunidades es otra de las funciones que aporta este servicio de mensajería.

\subsection{Radio digital y radio hertziana}

Las radios digitales pueden alcanzar a un tipo de oyente que no tiene los conocimientos necesarios o no pretende entrar en la dinámica canónica de la escucha de podcasts anteriormente descrita (descarga de una aplicación de gestión de podcasts, búsqueda de programas y posterior suscripción) y que, por tanto, le resulta más sencillo consumir estos contenidos de una forma más convencional -aunque en un soporte digital- bien en su ordenador o a través de dispositivos móviles. En este sentido, la emisora digital Radio Podcastellano juega un rol fundamental dentro de la podcastfera, al ser un medio centrado exclusivamente en la emisión de aquellos podcasts que solicitan admisión en su parrilla y pagan una pequeña cuota para el sustento de este medio en la Red.

Asimismo, la inclusión de ciertos espacios inicialmente construidos como podcasts en pequeñas emisoras de radio clásicas mantiene la misma lógica que observamos en el uso de la radio digital como soporte y punto de acceso diferenciado al contenido sonoro y de promoción de los proyectos. Existen, sin embargo, dos barreras que dificultan de forma decisiva la inserción de podcasts en radios hertzianas:

- la necesidad de periodicidad fija que obliga la radio tradicional, algo difícil de asumir para productores mediáticos 
amateurs cuya cadencia de grabación se puede ver afectada por motivos personales y laborales;

- la presencia de marcadas líneas editoriales o ideológicas en tales cadenas de radio.

A fin de preservar su independencia, los podcasters suelen evitar que sus programas queden "contaminados" por la línea ideológica de este tipo de emisoras. Afirma Goyo Salduero:

"A nosotros nos propusieron incluir nuestro programa en una radio analógica, pero dijimos que no porque no podemos garantizar una periodicidad estable".

\subsection{Programas presenciales en directo}

La realización de programas en directo en eventos destacados como las Jornadas $\mathrm{Na}$ cionales de Podcasting se considera como una práctica que extiende los mensajes del podcasting más allá de su lógica de consumo tradicional. La ejecución de estos espacios supone una práctica diferenciada con respecto a la dinámica habitual del medio ya que estos programas en directo no sólo se comparten en el mundo digital (después de ser grabados), sino que también se asientan en el terreno analógico en una interfaz diferente a las que se utilizan en el ciberespacio. Acudir como público a un podcast en directo implica una interacción directa y personal con el programa. El principal objetivo de la realización de estos espacios presenciales es el incremento del grado de popularidad de los podcasts, dada la gran cantidad de asistentes que estos eventos suelen reunir. En palabras de Esteban Pérez:

"Yo los veo más útiles para realizar relaciones personales que para el programa en sí".

Desde el lado de los oyentes, lo más interesante de esta manera de consumir los contenidos de los podcasts es la posibilidad de interacción usuario-creador en el plano físico y el hecho de que estos programas suelen ser significativamente diferentes con respecto a los programas habituales grabados y alojados online:

"A mí me gustan mucho, pues son oportunidades para ver a tus podcasters favoritos en programas que normalmente son distintos a los habituales" (oyente 3 ).

Las extensiones textuales amplían el contenido central del relato ofreciendo datos adicionales a lo ya conocido, funcionando como "nuevos capítulos" dentro de la narrativa de los podcasts

\subsection{Canales de vídeo digital}

Existen programas con cuentas propias en servicios de producción y alojamiento de vídeo digital. Estas plataformas se utilizan para desbordar los evidentes límites que tiene el lenguaje sonoro. Las principales aplicaciones de estos canales de vídeo -YouTube y Vimeo son los servicios de referencia en esta categoría- consisten en:

- realización de avances de los nuevos capítulos del podcast; - elaboración de anuncios de eventos especiales relacionados con el proyecto;

- incorporación del audio de los podcasts con una imagen estática;

- grabación en vídeo de la realización de algún episodio del podcast (sobre todo los grabados en grandes eventos o con motivo de ocasiones especiales);

- el registro audiovisual de los sorteos vinculados con los concursos que muchos de estos programas organizan para buscar la participación activa y la inmersión de los fans.

\subsection{Newsletters}

La generación de un amplio rango de contenidos por parte de determinados podcasts $y$, sobre todo, la creciente creación de redes de podcasts desde la esfera amateur, han potenciado el desarrollo de las newsletters distribuidas mediante correo electrónico como una forma de reunir de manera periódica el abundante material que generan los programas para todos aquellos usuarios suscritos. El consumo en movilidad que caracteriza al podcasting dificulta en ocasiones la consulta simultánea y en profundidad de determinadas informaciones relevantes incluidas en los podcasts, por lo que el recurso de la newsletter provee los enlaces a estas informaciones para su revisión en cualquier momento. Retomando la idea de McLuhan y Fiore (1967) acerca de los medios como ampliaciones del cuerpo humano, las newsletters vendrían a corregir parcialmente una de las grandes 
limitaciones que tiene el lenguaje sonoro del podcasting que, al igual que sucede con la radio, presenta una gran fugacidad.

\section{Taxonomía de los contenidos}

Situamos nuestro análisis en los contenidos que podemos considerar más relevantes para la narrativa del podcasting, aquellos que aportan matices o informaciones nuevas a los universos transmedia de los podcasts. Estas textualidades son: los contenidos adaptados y los contenidos expandidos.

\subsection{Contenidos adaptados}

Definidos como textos que manipulan, reorganizan, extractan, reutilizan o adaptan el contenido fundamental del programa (es decir, la generación de contenido a partir de variaciones de materiales preexistentes), estas textualidades pueden resultar útiles para recapitular o resumir los elementos más interesantes de las historias, si bien no constituyen extensiones en los relatos (Rodríguez-Ferrándiz; Ortiz-Gordo; Sáez-Núñez, 2014). En este sentido, estas textualidades han de ser consideradas como meras compresiones narrativas a las que recurren los podcasters transmedia para resumir o avanzar contenidos de sus podcasts, utilizando para ello diferentes plataformas y lenguajes mediáticos. Estas textualidades pueden ser:

- Resúmenes de los episodios: habitualmente se incluyen en los blogs o webs del proyecto. Cada episodio nuevo del podcast lleva vinculada una nueva entrada en tales webs que incluye datos resumidos sobre su contenido y enlaces a espacios online que amplían la información que se ofrece en el programa. Los proyectos que disponen de aplicación para dispositivos móviles utilizan la misma es-

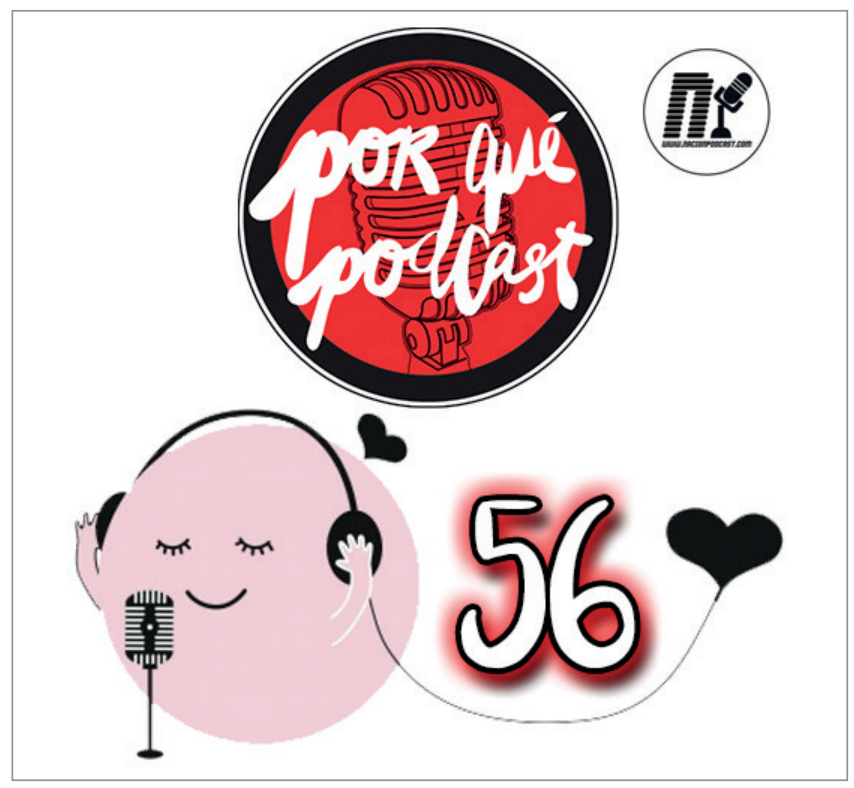

Carátula del episodio 56 del programa Porqué podcast http://nacionpodcast.com/wp-content/uploads/2017/08/56.jpg

trategia: cada nuevo episodio lleva aparejado un resumen de su contenido y los mismos enlaces en tal aplicación.

- Promos del podcast: constituyen píldoras mediático-promocionales creadas en formato sonoro a modo de cuña radiofónica y cuyo fin es dar a conocer el programa. Suelen ser de corta duración (no más de 1 minuto) y aparecen insertadas en los programas de otros podcasters de forma gratuita, generando así estrategias de promoción cruzada.

- Avances de capítulos: el avance de nuevos contenidos ha sido una estrategia ampliamente utilizada en los medios

Tabla 3. Distribución funcional de las plataformas utilizadas por el podcasting transmedia. Entre paréntesis se indica el porcentaje de podcasts de la muestra que utiliza cada plataforma.

\begin{tabular}{|c|c|c|}
\hline Plataforma & $\begin{array}{l}\text { \% de uso entre los pro- } \\
\text { gramas analizados }\end{array}$ & Funciones \\
\hline Plataformas y podcatchers & 100 & $\begin{array}{l}\text { Alojamiento inicial de todo podcast. } \\
\text { Construcción de comunidades. } \\
\text { Puerta de acceso principal a los proyectos. }\end{array}$ \\
\hline Web/blog & 100 & $\begin{array}{l}\text { Expansión narrativa para completar/complementar contenido sonoro. } \\
\text { Principal puerta de acceso al proyecto desde buscadores. } \\
\text { Convergencia mediática. Reunión de todos los contenidos del proyecto. } \\
\text { Determina la personalidad del programa. } \\
\text { Punto de acceso sencillo para usuarios menos tecnológicos. }\end{array}$ \\
\hline Redes sociales & 100 & $\begin{array}{l}\text { Interacción y diálogo con fans. } \\
\text { Generación de contenido. } \\
\text { Captación de nuevos oyentes (promoción). } \\
\text { Participación del usuario menos comprometido. }\end{array}$ \\
\hline Radio digital y hertziana & 72,7 & Punto de acceso diferenciado al contenido del podcast. \\
\hline Programas presenciales & 36,4 & $\begin{array}{l}\text { Conexión personal entre podcasters y oyentes. } \\
\text { Valor promocional. }\end{array}$ \\
\hline Canales de vídeo digital & 36,4 & $\begin{array}{l}\text { Grabación de capítulos especiales. } \\
\text { Avances de próximos capítulos. }\end{array}$ \\
\hline Newsletters & 9,1 & Reunión de los contenidos de los proyectos. \\
\hline
\end{tabular}


de masas, especialmente en la televisión. Reproduciendo esta dinámica, muchos podcasts realizan grabaciones tanto en formato sonoro como audiovisual que contienen los aspectos fundamentales que serán tratados en próximos episodios. Estos avances son más utilizados por los espacios que tienen una frecuencia inferior a la quincenal.

\subsection{Contenidos expandidos}

Esta categoría incluye todas las textualidades que, en lugar de comportarse como compresiones narrativas, proveen información novedosa que expande el contenido presentado en el podcast. Hay dos subcategorías: extensiones textuales e información metatextual.

\section{Extensiones textuales}

Amplían el contenido central del relato ofreciendo datos adicionales a lo ya conocido, funcionando como nuevos capítulos dentro de la narrativa de los podcasts. Podemos encontrar:

- Noticias temáticas: todas las informaciones relacionadas con la temática del programa que, de elaboración propia o construidas por terceros, el podcaster provee a sus seguidores en las diferentes plataformas del proyecto.

- Vídeos con contenido diferenciado: material presentado en formato audiovisual que ofrece informaciones no tratadas en el podcast. Veamos algunos ejemplos. Numerosos programas dedicados a la tecnología utilizan su canal de YouTube para realizar tutoriales o revisiones sobre determinados productos de software o nuevos dispositivos aprovechando las posibilidades del lenguaje audiovisual. Estos contenidos tienen difícil cabida en un podcast dadas las limitaciones del lenguaje sonoro. Otros programas alojan vídeos en sus canales para realizar los sorteos de determinados concursos, así como debates entre varios de los miembros del equipo del programa, cursos formativos o coberturas de eventos en directo. Incluso, encontramos ejemplos de podcasts que amplían sus contenidos a través del lanzamiento de miniseries de animación 2D en sus canales de vídeo de YouTube o Vimeo.

- Contenido multiprograma y spin-off: ambas estrategias consisten en la creación de nuevos podcasts derivados de un programa matriz a fin de aumentar la variedad de contenidos ofrecidos a sus seguidores y fans. La estrategia multiprograma es aquella en la que los productores realizan espacios del mismo tema que el podcast nodriza, pero con un formato o duración diferente. Suelen ser espacios más breves y específicos dedicados a la ampliación o profundización de un tema más concreto del que suele aparecer en los espacios padre, que tienen mayor duración y un carácter más general. El modelo spin-off es sensiblemente distinto: los programas derivados suelen presentar mayor distancia con respecto al podcast matriz desde el punto de vista temático, incluso pueden estar presentados por locutores diferentes y presentar suscripción diferenciada.

- Contenidos off-topic: proyectos de podcasting que, al margen de la web central, construyen un segundo espacio online habitualmente en formato blog que en ocasiones se utiliza para incluir asuntos off-topic no relacionados con el tema general del proyecto.
- Otras textualidades: si bien los contenidos anteriormente indicados son los más utilizados, encontramos otros textos que, de forma menos profusa, también son utilizados en este tipo de proyectos: la publicación de libros digitales y en papel que complementan los contenidos del podcast y la realización de carátulas específicas en formato visual -con entidad narrativa autónoma- para cada capítulo del programa son los más habituales.

La aparición del podcasting y su posterior evolución transmedia ha generado nuevas posibilidades narrativas tras un amplio período en el que la comunicación sonora fue dominada por una radio anclada en una preocupante estandarización de géneros y formatos

\section{Información metatextual}

Es información sobre el propio proyecto, sus creadores, la marca o su mundo narrativo ofreciendo datos nuevos no conocidos pero sin ampliar el contenido de los relatos previamente narrados. Se establecen cuatro subcategorías:

- Información industrial: información que ofrece el proyecto para dar a conocer el proceso creativo o constructivo de los contenidos; es decir, el sujeto de la información es el desarrollo productivo en sí mismo. En este subtipo, podemos encontrar el making-of del podcast y del resto de textualidades que extienden su relato (vídeos, libros, etc.).

- Información sobre los autores: datos de los productores del proyecto y de los colaboradores puntuales o entrevistados que participan esporádicamente o circunstancialmente en alguno de los episodios del podcast. En este caso, el sujeto de la información es el creador/colaborador/invitado del programa, cuyos datos suelen ser alojados en el sitio online del proyecto.

- Información sobre la marca: el sujeto de la información es el proyecto como entidad, la marca o su mundo narrativo. Incluimos en esta clase a todos los anuncios sobre la organización y/o participación del podcast (y/o sus representantes) en eventos, así como la información de su experiencia en éstos. También agrupamos en esta categoría las noticias generadas por la actividad del programa: nominaciones a premios, galardones, recomendaciones por parte de terceros, incorporación de nuevos miembros, grabación de nuevos formatos y cualquier información vinculada a la actividad general del proyecto. Estos contenidos suelen tener carácter multiplataforma al encontrarse alojados tanto en las web o blogs, las redes sociales y en pequeñas secciones del contenido sonoro publicado.

- Información instrumental: son los elementos comunicativos que ofrecen datos sobre el programa y, a la vez, sirven para facilitar la interacción y el consumo de los contenidos. Un ejemplo es la inserción del minutado de las diferentes secciones que podemos encontrar en los podcasts, de forma que el usuario es guiado por los distintos contenidos tratados en un capítulo a fin de buscar los aspectos 
Tabla 4. Taxonomía de los contenidos utilizados por el podcasting transmedia. Se indica el porcentaje de podcasts de la muestra que incluye cada tipo de contenido

\begin{tabular}{|c|c|c|c|}
\hline \multicolumn{2}{|c|}{ Contenidos adaptados } & \multicolumn{2}{|l|}{ Contenidos expandidos } \\
\hline \multirow[b]{2}{*}{ Resúmenes de episodios } & \multirow{2}{*}{$\begin{array}{c}\% \\
90,9\end{array}$} & Extensiones textuales & $\%$ \\
\hline & & Noticias temáticas & 27,3 \\
\hline \multirow{2}{*}{ Promos del podcast } & \multirow{2}{*}{54,5} & Vídeos con contenido diferenciado & 27,3 \\
\hline & & Contenido multiprograma y spin-off & 36,4 \\
\hline \multirow{2}{*}{ Avances de capítulos } & \multirow{2}{*}{9,1} & Material off-topic & 9,1 \\
\hline & & Carátulas & 9,1 \\
\hline & & Información metatextual & $\%$ \\
\hline & & Información industrial & 36,4 \\
\hline & & Información sobre los autores & 27,3 \\
\hline & & Información sobre la marca & 45,5 \\
\hline & & Información instrumental & 9,1 \\
\hline
\end{tabular}

del contenido sonoro, funcionan como constructores de la personalidad e identidad del proyecto y facilitan la monitorización de sus seguidores a través de los datos generados por sus patrones de navegación.

En las redes sociales, los podcasts logran altas posibilidades de interacción, diálogo y participación con sus fans y facilitan, a su vez, la construcción de contenidos significativos para el podcast. Las redes sociales constituyen, por último, una interesante herramienta promocional capaz de captar a nuevos oyentes. más interesantes y facilitar una escucha no lineal de los mismos. Esta información metatextual, incluida tanto en los sitios online como en los metadatos que acompañan a los podcasts en los servicios de alojamiento, ofrece datos sobre el propio programa (qué asuntos se incluyeron en cada una de las secciones, cuántas secciones tiene un programa, cuál es su orden y duración, etc...) y, a la vez, sirve como instrumento que facilita la relación del usuario con el programa, presentando así una doble capacidad informativa e interactiva. El programa es sujeto de la información y, a la vez, objeto de la acción que permite el instrumento referido.

\section{Conclusiones}

La narrativa actual del podcasting desborda el carácter exclusivamente sonoro de sus inicios al construir productos mediáticos y culturales multilenguaje. Si bien el objeto nuclear de estos trabajos continúa siendo el contenido sonoro, los podcasters amueblan su universo narrativo con producciones que utilizan otros formatos mediáticos. El uso de fotografías, texto y recursos audiovisuales contribuyen a extender, comprimir o repetir los contenidos que provee este medio.

Los podcasts utilizan una amplia variedad de plataformas tanto online como offline. Aunque destaca sobremanera el uso de las plataformas digitales, numerosos proyectos cuentan con un importante soporte fuera del mundo virtual, en radios hertzianas y a partir de la producción de programas presenciales en directo. Además de servir como punto de acceso diferenciado al universo narrativo, cada una de las plataformas utilizadas aporta valores específicos al conjunto del proyecto, estableciéndose un proceso de diferenciación funcional que hace que cada plataforma explote sus fortalezas de la forma más efectiva posible. Por ejemplo, las webs o blogs sirven para desarrollar expansiones narrativas a modo de complemento
El podcasting ofrece una multiplicidad de contenidos que, lejos de comportarse de forma aislada e inconexa, guardan conexiones entre sí desarrollando una gramática propia, coherente y lógica. Los resúmenes de los episodios, las promos de los podcasts a modo de cuñas publicitarias y los avances de capítulos son ejemplos de compresiones narrativas que gravitan en torno al contenido de los programas. Existen, a la vez, otros textos que sí extienden la narrativa de los espacios ofreciendo nuevos datos que no podemos encontrar en la grabación sonora. Claros ejemplos de estas extensiones narrativas son:

- las noticias relacionadas con el tema sobre el que versa el podcast que suelen aparecer en las webs del proyecto;

- los vídeos con contenido diferenciado;

- las estrategias multiprograma y spin-off.

En algún punto situado entre los contenidos redundantes y las extensiones narrativas, encontramos los metatextos, los

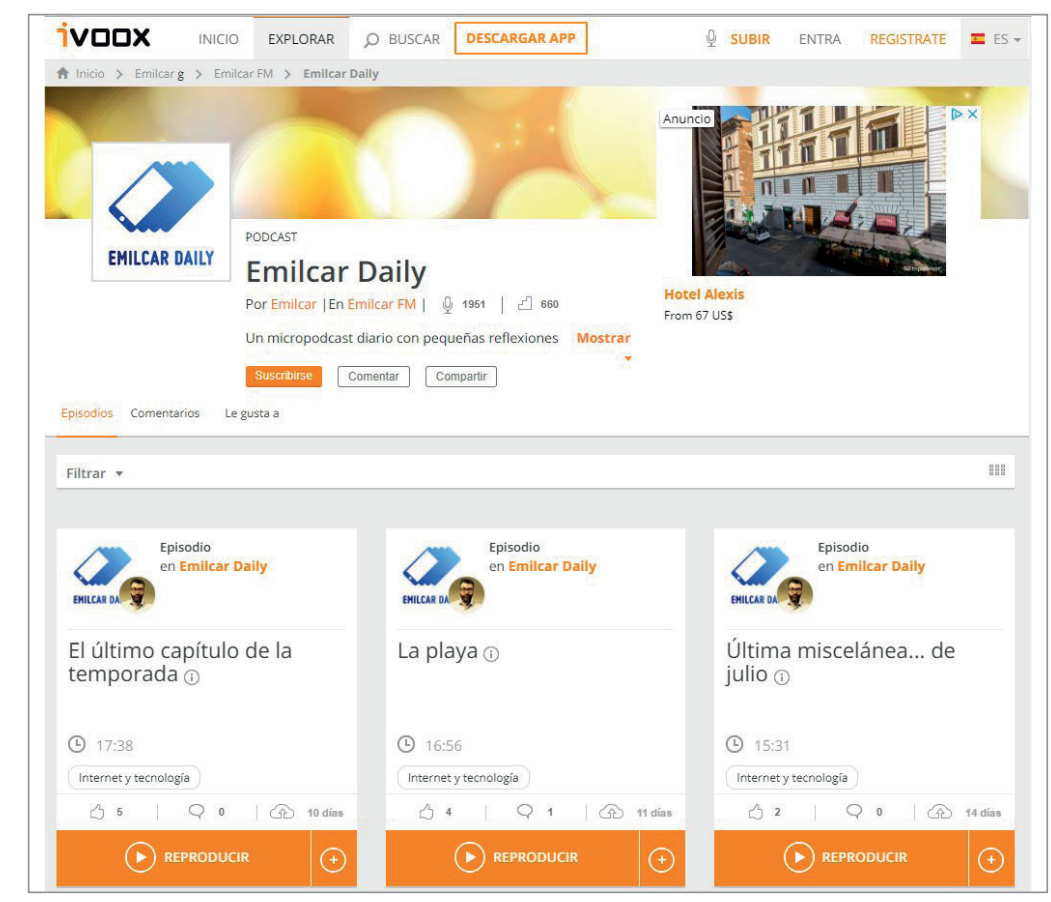

Canal del podcast independiente Emilcar Daily en el servicio de alojamiento iVoox https://www.ivoox.com/podcast-emilcar-daily_sq_f145026_1.html 
elementos narrativos que no amplían el relato y, a la vez, ofrecen contenidos nuevos relacionados con los procesos creativos de los programas (making-of), con la propia marca como objeto de la información y con sus autores.

La aparición del podcasting, con su posterior evolución transmedia, ha generado nuevas posibilidades narrativas tras un amplio período en el que la comunicación sonora fue dominada por la radio que, durante muchas décadas, permaneció anclada en una preocupante estandarización de géneros y formatos. El podcasting ha alimentado una nueva forma de construir productos sonoros a partir de la adición de múltiples manifestaciones expresivas que se dispersan en espacios diversos, tanto digitales como analógicos. Su lenguaje constituye una verdadera ruptura frente al modelo exclusivamente sonoro de sus inicios y frente a las lógicas narrativas de la radio tradicional.

\section{Agradecimientos}

Este trabajo se ha elaborado en el marco de la Red $1+D$ de Educación Mediática del Programa Estatal de Investigación Científica-Técnica de Excelencia, Subprograma Estatal de Generación de Conocimiento (EDU2016-81772-REDT), financiados por el Fondo Europeo de Desarrollo Regional (Feder) y Ministerio de Economía y Competitividad de España (Mineco).

\section{Referencias}

Aparici, Roberto; García-Marín, David (2018). "Prosumidores y emirecs: análisis de dos teorías enfrentadas". Comunicar, v. 26, n. 55, pp. 71-79.

https://doi.org/10.3916/C55-2018-07

Askwith, Ivan (2017). "Television 2.0: Reconceptualizing TV as an engagement medium". Comparative media studies, August $10^{\text {th }}$.

http://cmsw.mit.edu/television-2-0-tv-as-an-engagementmedium

Berry, Richard (2016). "Will the iPod kill the radio star? Profiling podcasting as radio". Convergence: The international journal of research into new media technologies, v. 12, n. 2, pp. 143-162.

https://bit.ly/2LgeJCJ

https://doi.org/10.1177/1354856506066522

Bonini, Tiziano (2015). "The second age of podcasting: reframing podcasting as a new digital mass medium". Cuaderns del CAC, v. 18, n. 41, pp. 21-30.

https://bit.ly/2LhLyz5

Callejo, Javier; Viedma, Antonio (2005). Proyectos y estrategias de investigación social: la perspectiva de la intervención. Madrid: McGraw Hill/Interamericana de España. ISBN: 9788448146139

Cebrián-Herreros, Mariano (2009). La radio en la convergencia multimedia. Barcelona: Gedisa. ISBN: 97884743 28929

Corbetta, Piergiorgio (2007). Metodologías y técnicas de investigación social. Madrid: McGraw Hill/Interamericana de España. ISBN: 9788448156107
Dubber, Andrew (2013). Radio in the digital age. Cambridge, UK: Polity Books. ISBN: 9780745661971

Gallego-Pérez, Juan-Ignacio (2010). Podcasting: distribución de contenidos sonoros y nuevas formas de negocio en la empresa radiofónica española. Tesis doctoral. Universidad Complutense de Madrid.

http://eprints.ucm.es/11205/1/T32070.pdf

García-Marín, David (2017). “La nueva comunicación sonora. Del podcast al transcasting". En: Aparici, Roberto; García-Marín, David. iSonríe, te están puntuando! Narrativa digital interactiva en la era de Black Mirror. Barcelona: Gedisa, pp. 145-163. ISBN: 9788416919734

Jenkins, Henry (2006). Convergence culture. La cultura de la convergencia en los medios de comunicación. Barcelona: Paidós. ISBN: 9788449321535

https://bit.ly/1iFsTzb

Massarelli, Virginia; Perrotta, Marta (2006). "Podcasting: A change for listeners, a challenge for broadcasters". In: Thiermann, Sven (ed.). Relating radio. communities. Aesthetics. Access. Leipzig: Spector, pp. 267-264.

McHugh, Siobhan (2016). "Oral history and the radio documentary/feature: Introducing the Cohrd form". Radio journal: International studies in broadcast \& audio media, v. 10, n. 1, pp. 35-51.

https://bit.ly/2NaS7W2

McLuhan, Marshall; Fiore, Quentin (1967). El medio es el masaje. Un inventario de efectos. Barcelona: Paidós. ISBN: $847509015 \mathrm{X}$

Moreno, Isidro (2012). La convergencia interactiva de medios: hacia la narración hipermedia. Tesis doctoral. Universidad Complutense de Madrid.

http://eprints.ucm.es/17217/1/T20881.pdf

Ortiz-Sobrino, Miguel-Ángel (2012). "Radio y post-radio en España: una cohabitación necesaria y posible". Área abierta, v. 12, n. 2.

http://revistas.ucm.es/index.php/ARAB/article/view/39637

Rodríguez-Ferrándiz, Raúl; Ortiz-Gordo, Félix; Sáez-Núñez, Virginia (2014). "Contenidos transmedia de las teleseries españolas: clasificación, análisis y panorama en 2013". Communication \& society, v. 27, n. 4, pp. 73-94.

https://www.unav.es/fcom/communication-society/es/ articulo.php?art_id $=513$

Rosado-Millán, María-Jesús; García-García, Francisco; Kaplún-Hirsz, Daniel (2014). Guía práctica de técnicas de investigación social. Madrid: Fundación iS+D para la Investigación Social Avanzada. ISBN: 9788494051333

Scolari, Carlos A. (2008). Hipermediaciones. Elementos para una teoría de la comunicación digital interactiva. Barcelona: Gedisa. ISBN: 9788497842730

Wired (2004). "Podcasts: new twist on net audio". Wired, August $10^{\text {th }}$.

http://archive.wired.com/entertainment/music/ news/2004/10/65237 